\title{
Improvement of erectile function by Korean red ginseng (Panax ginseng) in a male rat model of metabolic syndrome
}

\author{
Sung-Dae Kim ${ }^{1}$, Young-Joo Kim ${ }^{1}$, Jung-Sik Huh ${ }^{1}$, Sae-Woong Kim ${ }^{2}$ and Dong-Wan Sohn ${ }^{2}$
}

The seriousness of metabolic syndrome is not due to the disease itself but its promotion of other diseases, such as erectile dysfunction and cardiovascular and cerebrovascular diseases. We investigated the effects of Korean red ginseng (KRG, Panax ginseng) extract on erectile function in a rat model of metabolic syndrome. We divided the rats into three groups: control, metabolic syndrome+normal saline (N/S) and metabolic syndrome+KRG. To determine the occurrence of metabolic syndrome in all groups, body weight and various biochemical parameters (e.g., blood glucose, insulin, cholesterol) were measured, and the intra-abdominal glucose tolerance test was performed. To investigate penile erection, the peak intracavernosal pressure (ICP), mean arterial pressure (MAP) and Masson's trichrome stain were evaluated. Erectile function was also investigated by measuring the cyclic guanosine monophosphate (cGMP) levels of the corpus cavernosum. We found that the various biochemical parameters and body weight were similar in the metabolic syndrome +KRG group and the control group, although the values were slightly higher. The peak ICP/MAP ratio of the metabolic syndrome $+\mathrm{N} / \mathrm{S}$ group was markedly decreased compared to the other groups. The cGMP level of the corpus cavernosum in the metabolic syndrome+N/S group was significantly lower than that of the other groups. As demonstrated in this model of metabolic syndrome with erectile dysfunction, KRG may improve erectile function.

Asian Journal of Andrology (2013) 15, 395-399; doi:10.1038/aja.2012.159; published online 4 February 2013

Keywords: erectile dysfunction; ginseng; metabolic syndrome; penis

\section{INTRODUCTION}

Due to the rapid transition to an aging society and enhanced quality of life in Korea, erectile dysfunction has become one of the most common diseases in middle-aged and elderly males. The pathophysiological mechanisms of erectile dysfunction are very diverse, and the causes of the disease include dysfunction of the cavernous nerves of the penis and endothelial cells, smooth muscle fibrosis, reduced nitric oxide production and reduced cyclic guanosine monophosphate (cGMP), which is a secondary messenger. ${ }^{1,2}$

The definitions of metabolic syndrome are heterogeneous because multiple sets of diagnostic criteria were initially created to identify insulin-resistant subjects or to predict clinical events, such as cardiovascular disease. At present, metabolic syndrome is a combination of symptoms composed of cardiovascular risk factors such as central abdominal obesity, hypertension, high fasting blood glucose and dyslipidemia, such as hypertriglyceridemia. ${ }^{3}$ The seriousness of metabolic syndrome is not only due to the disease itself but also its promotion of other diseases, such as erectile dysfunction and cardiovascular and cerebrovascular diseases. The prevalence of erectile dysfunction in metabolic syndrome patients has been reported to be twofold greater than control patients. Greater numbers of risk factors of metabolic syndrome increase the risk of erectile dysfunction. ${ }^{4}$ In addition, meta- bolic syndrome has been associated with lower urinary tract symptoms, prostate cancer and the formation of urinary stones. ${ }^{5,6}$ In Korea, most studies on metabolic syndrome have been conducted by internists and focus on the relationships with cardiovascular disease. Few study outcomes have been reported in the field of urology in Korea. In our previous study, we developed a rat model of metabolic syndrome to study the effects of metabolic syndrome on penile erectile function and the corpus cavernosum penis. ${ }^{7}$

Korean red ginseng (KRG, Panax ginseng) is a representative herbal medicine used for many years in the treatment and prevention of diseases. Red ginseng is Panax ginseng that has been heated either through steaming or sun drying. Based on previous studies, we evaluated the effects of KRG on the improvement of blood biochemical parameters associated with metabolic syndrome, erectile function and restoration of the corpus cavernosum in a rat model of metabolic syndrome.

\section{MATERIALS AND METHODS}

\section{Animal experiments and diet}

White male Wistar-Kyoto rats were used as the control group (group I, $n=10$ ). The experimental groups included 7-week-old rats with spontaneous hypertension (SHR) divided into group II $(n=10)$ and group III $(n=10)$. Group I was fed a normal-fat diet (fat 16.4\%) and normal 
saline every day. Group II was fed a high-fat diet (fat 45\%) and normal saline every day, and group III was fed a high-fat diet and $100 \mathrm{mg}$ of KRG extract dissolved in $2 \mathrm{ml}$ of distilled water through a gastric tube every day for 12 weeks. Cheong-Kwan-Jang KRG extract powder (6year-old red ginseng; Korea Ginseng Corporation, Seoul, Korea) was used as the KRG extract. The body weight of each animal was measured every 4 weeks. Systolic blood pressure was measured at the tail of the rats using a tail-cuff system (IITC Life Science, Woodland Hills, CA, USA). At the twelfth week of the experiment, an intraperitoneal glucose tolerance test was conducted by administering $50 \% \mathrm{D} / \mathrm{W}$ glucose via an intra-abdominal injection at $30 \mathrm{mg} \mathrm{kg}^{-1}$, and a blood sample was collected from the tail at 30, 60, 90 and $120 \mathrm{~min}$.

\section{Intracavernosal pressure (ICP) measurement}

The rat penis was detached with the rat in a supine position to expose the corpus cavernosum penis and the crus of the penis, and then the pelvic ganglia, located at the lateral prostate gland, were detached and exposed through a median abdominal incision. The right internal carotid artery was detached before intubation with a $24 \mathrm{G}$ polyethylene catheter to measure mean arterial pressure (MAP). To measure ICP, a 23G scalp vein needle was indwelled in the corpus cavernosum penis, and a bipolar electric stimulator was positioned at the detached ganglia before gradually increasing the frequency to $2.5,5,10$ and $20 \mathrm{~Hz}$ over a 50 -s period under the following conditions: $10 \mathrm{~V}, 2.4 \mathrm{~mA}$ and $0.5 \mathrm{~ms}$ square wave duration. A nerve conduction study was conducted on the corpus cavernosum penis at least three times, and the nerve impulse interval was more than $10 \mathrm{~min}$. Two corpus cavernosum penises were extracted; one was stored in liquid nitrogen, and the other was fixed in formalin.

\section{Biochemical indicators}

After measuring the ICP, the rats were killed, and a blood sample was obtained from the inferior vena cava. For the intraperitoneal glucose tolerance test, an ACCU-CHEK glucometer (Roche Diagnostics, St Louis, MO, USA) was used. The total cholesterol concentration was measured using an ADVIA 1650 system (Bayer, Tarrytown, NY, USA), and the free fatty acid concentration was measured through an enzyme-based method using acyl-CoA synthetase. Plasma renin activity and the serum aldosterone concentration were measured using a radioimmunoassay. The blood insulin concentration was measured using an insulin ELISA kit (Shibayagi, Ishihara, Japan). Insulin resistance was assessed with the HOMA-IR (HOMA insulin resistance index), which is frequently used in endocrinology. ${ }^{8}$

HOMA insulin resistance index $=$ fasting insulin $\left(\mu \mathrm{U} \mathrm{ml}^{-1}\right) \times$ fasting blood sugar $\left(\mathrm{mmol} \mathrm{l}^{-1}\right) / 22.5$.

\section{Tissue staining and histomorphological analysis}

Fixed histological sections of the corpus cavernosum penis were washed with phosphate-buffered saline (PBS) and then dehydrated with ethanol in increasing concentrations before preparing paraffin sections. The sections were sliced at a thickness of $6 \mu \mathrm{m}$. The slices were mounted on gelatine-coated glass slides and were observed before staining with Masson's trichrome. The slides stained with Masson's trichrome were analysed using a computer-aided histomorphological image analyser (Image-Pro Plus, version 5.0; Media Cybernetics, Bethesda, MD, USA) at $\times 200$ magnification. The mean ratio of smooth muscle cells to total cells in the corpus cavernosum of penis tissue was calculated from 15 high-power fields.

\section{Immunohistochemistry for TGF- $\beta_{1}$}

Immunohistochemical staining for TGF- $\beta$ immunoreactivity was performed using a Vector ABC kit (Vector Laboratories, Burlingame, CA,
USA). Monoclonal mouse anti-TGF- $\beta_{1}$ (Biosource International, Nivelles, Belgium) was used as the primary antibody. After deparaffinisation using xylene and decreasing ethanol concentrations, endogenous peroxidase activity was quenched in a mixture of $60 \%$ methanol and $3 \% \mathrm{H}_{2} \mathrm{O}_{2}$ for $30 \mathrm{~min}$. The sections were then washed with PBS and reacted with $5 \%$ normal horse serum for $1 \mathrm{~h}$ to inhibit nonspecific responses in the peripheral area before incubation with the TGF- $\beta_{1}$ polyclonal antibody at $4{ }^{\circ} \mathrm{C}$ for $16-18 \mathrm{~h}$. In control sections, normal horse serum was used instead of the primary antibody. The stained sections were washed with PBS and then incubated with biotinylated anti-mouse IgG for $30 \mathrm{~min}$. The sections were incubated with avidin and biotinylated horseradish peroxidase for $30 \mathrm{~min}$ and washed with PBS. Colour was developed using 3,3-diaminobenzidine tetrahydrochloride for $7 \mathrm{~min}$, and the sections were dehydrated and coverslipped using Permount before observing them with a light microscope.

\section{Measurement of the cGMP level in smooth muscles in the corpus} cavernosum penis

To measure the cGMP level, a commercial cGMP $\left[{ }^{125} \mathrm{I}\right]$ radioimmunoassay kit (Amersham, Buckinghamshire, UK) was used. The idea of the experiment was to induce a competitive fixation reaction between ${ }^{125}$ I-labelled cGMP, which was present in a fixed amount, and cGMP, which was present in the cells. The isotope weights of ${ }^{125}$ I-labelled cGMP were then measured to determine the cGMP concentration in the samples. Preliminary experiments demonstrated that the levels of cGMP in the tissues and smooth muscles of the corpus cavernosum penis were low, and an acetylation assay was therefore used. The dry extracts of the cytoplasm were dissolved in the assay buffer of the kit, and then, the acetylation reagent, antiserum and $\left[{ }^{125} \mathrm{I}\right] \mathrm{cGMP}$ were added to continue the reactions according to the kit protocol. Finally, the radioactivity (cpm) of each tube was measured using a gamma scintillation counter, and the cGMP concentration of each sample was calculated using the obtained standard curve.

\section{Statistical analysis}

SPSS software (version 12.0, Windows) was used for the statistical analyses. Statistical significance was examined using the MannWhitney test, which is a nonparametric method. At $P<0.05$, results were considered significant.

The study protocol was first approved by a central ethics committee (Jeju National University Hospital, Jeju, Korea; No. 2012-0003) and then by the respective local ethics committees.

\section{RESULTS}

\section{Body weight, blood pressure and biochemical indexes}

To confirm the development of metabolic syndrome, body weight, blood pressure and the biochemical index of each group were measured (Table 1). The body weight of group III was slightly greater than that of group I, but the difference was not significant (Figure 1a). The change of body weight and systolic blood pressure of group II in 12week increased significantly compared to group I $(P=0.010$ and $P=0.014$, respectively) (Figure 1a, $1 \mathbf{b}$ ).

\section{ICP changes}

The peak ICP was $82.3 \pm 4.6,60.5 \pm 6.5$ and $68.8 \pm 5.4 \mathrm{mmHg}$ in group I, group II and group III, respectively. Group II had the lowest level, but the difference was not statistically significant. The peak ICP/MAP ratios were $53.0 \% \pm 6.5 \%, 32.0 \% \pm 4.5 \%$ and $49.0 \% \pm 4.5 \%$, respectively, with a slightly lower level in group III compared to group I, although the difference was not significant; the ratio in group II was significantly lower compared to group I $(P=0.016)$ (Figure 2). 
Table 1. Biochemical parameters of each group.

\begin{tabular}{lcccc}
\hline Parameter & Group I & Group II & Group III & $P$ \\
\hline Body weight $(\mathrm{g})$ & $420 \pm 22$ & $620 \pm 35$ & $450 \pm 55$ & 0.010 \\
systolic blood pressure $(\mathrm{mmHg})$ & $115 \pm 15$ & $170 \pm 15$ & $125 \pm 15$ & 0.014 \\
Total cholesterol (mg dl & -1 \\
Free fatty acid $\left(\mu \mathrm{Eq} \mathrm{l}^{-1}\right)$ & $56 \pm 5$ & $122 \pm 8$ & $62 \pm 5$ & 0.028 \\
Aldosterone $\left(\mathrm{pg} \mathrm{ml}^{-1)}\right.$ & $440 \pm 25$ & $780 \pm 65$ & $470 \pm 85$ & 0.018 \\
PRA $\left(\mathrm{ng} \mathrm{ml}^{-1} \mathrm{~h}^{-1}\right)$ & $315 \pm 73$ & $420 \pm 64$ & $340 \pm 68$ & 0.021 \\
Insulin $\left(\mu \mathrm{mll}^{-1}\right)$ & $7.2 \pm 0.5$ & $8.7 \pm 0.6$ & $8.25 \pm 0.6$ & $0.175 *$ \\
HOMA-IR & $2.30 \pm 0.45$ & $1.15 \pm 0.24$ & $2.05 \pm 0.32$ & 0.014 \\
\hline
\end{tabular}

Abbreviations: HOMA-IR, HOMA insulin resistance index; PRA, plasma renin activity.

Group I, control; Group II: metabolic syndrome + normal saline; Group III: metabolic syndrome + Korean red ginseng

*only not compared to difference in statistics

Distribution of smooth muscles of the corpus cavernosum penis The smooth muscle cells of the corpus cavernosum penis were observed using Masson's trichrome. In groups I and II, the smooth muscle cells were dyed dark brown, and the collagen fibres were dyed blue; both were regularly arranged. By comparison, the collagen fibres in group II were condensed and irregularly distributed. According to the histomorphological image analysis, the ratio of smooth muscle cells in the corpus cavernosum penis in group II was $10.8 \% \pm 3.0 \%$ and was significantly different compared to group I $(P=0.023)$ (Figure 3).

\section{Immunohistochemical changes}

Immunohistochemistry was performed to assess TGF- $\beta_{1}$ expression. Group I showed normal cells in the corpus cavernosum penis tissue sections with weak TGF- $\beta_{1}$ immunoreactivity, while group II showed strong TGF- $\beta_{1}$ immunoreactivity with increased expression in the
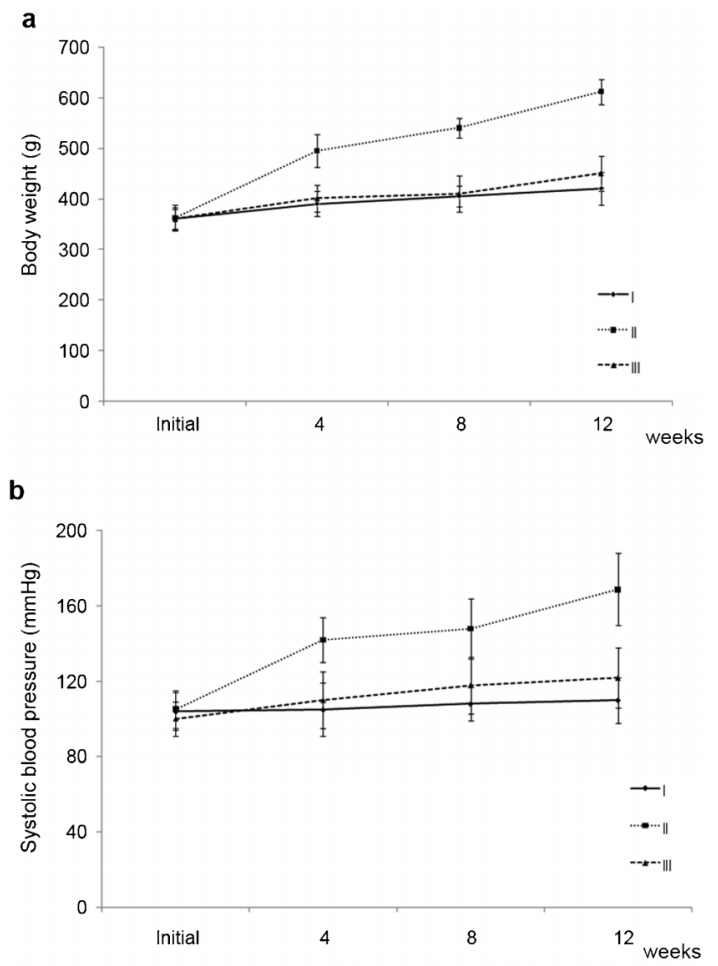

Figure 1 The change of body weight (a) and systolic blood pressure (b) in each group over the 12-week study period. While body weight $(P=0.010)$ and systolic blood pressure ( $P=0.014$ ) increased significantly in group II compared to group I, those of group III were similar to group I, although they were slightly higher. Group I, control; Group II: metabolic syndrome + normal saline; Group III: metabolic syndrome + Korean red ginseng. collagenous connective tissues and fibroblasts around the cavernosus. In addition, collagen was increased due to fibrosis. By contrast, group III, which was administered KRG, showed significantly decreased expression compared to group II (Figure 4).

Measurement of the cGMP level in the smooth muscles of the corpus cavernosum penis

To assess penile erectile function, the cGMP level was measured. Group

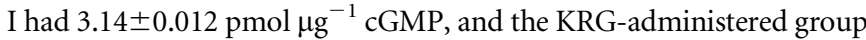

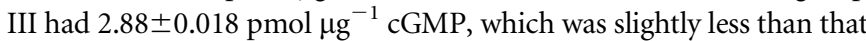
of the control group. Group II, which was administered normal saline,

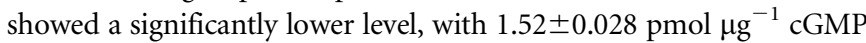
$(P=0.005)$ (Figure 5).

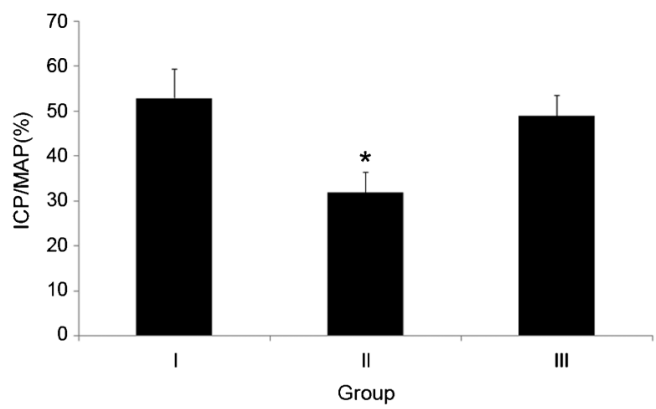

Figure 2 Comparison of the ratio of the peak ICP/MAP in each group. The ratio of peak ICP/MAP decreased significantly in group II ( $P=0.016$ vs. group I), whereas it was similar in group III and group I. ICP, intracavernosal pressure; MAP, mean arterial pressure. Group I, control; Group II: metabolic syndrome + normal saline; Group III: metabolic syndrome + Korean red ginseng.

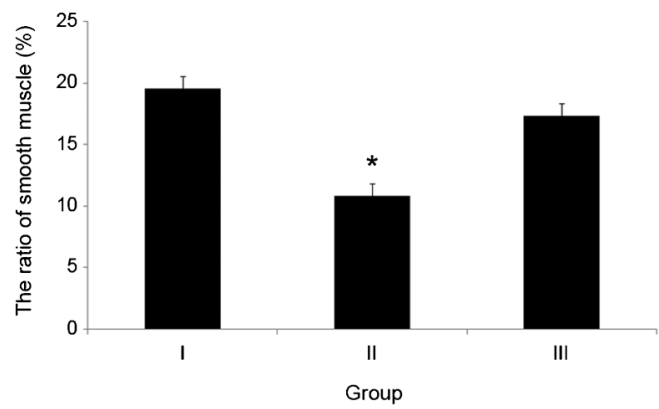

Figure 3 The distribution of smooth muscle observed using Masson's trichrome staining of the corpus cavernosum in each group. The ratio of smooth muscle in group II was significantly lower than the other groups ( $* P=0.023$ vs. group I), while it was similar in groups III and I. Group I, control; Group II: metabolic syndrome + normal saline; Group III: metabolic syndrome + Korean red ginseng. 


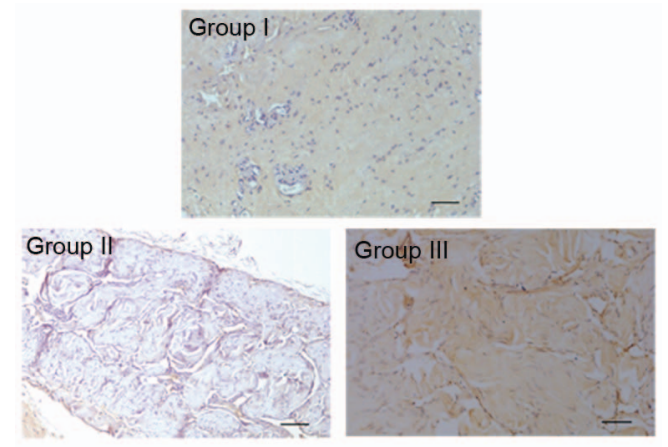

Figure 4 A comparison of the immunohistochemical expression of TGF- $\beta_{1}$ in each group. In group I, normal structures and weak immunoreactivity to TGF$\beta_{1}$ were observed in the corpus cavernosum tissue. By contrast, in group II, TGF$\beta_{1}$ immunoreactivity was strong and tended to increase in collagen, fibroblasts and smooth muscle fibres. In group III, TGF- $\beta_{1}$ immunoreactivity was decreased compared with group II. Scale bar $=100 \mu \mathrm{m}$. Group I, control; Group II: metabolic syndrome + normal saline; Group III: metabolic syndrome + Korean red ginseng.

\section{DISCUSSION}

According to a literature review of metabolic syndrome, it is closely related to erectile dysfunction and hypogonadism. Metabolic syndrome may lead to erectile dysfunction through multiple mechanisms, including hypogonadism, atherosclerosis, endothelial dysfunction, hyperglycaemia and urogenital sensory neuropathy. ${ }^{9}$ Corona et al. ${ }^{10}$ demonstrated that testosterone levels were inversely related to the severity of erectile dysfunction and directly related to the magnitude of penile blood flow in the oldest quartile of patients.

In particular, the cardiovascular risk factors of metabolic syndrome and erectile dysfunction show a high correlation. ${ }^{11}$ Metabolic syndrome is an aggregation of risk factors of coronary arteriosclerotic heart disease, including obesity, glucose intolerance, dyslipidaemia and hypertension. In addition, the prevalence of erectile dysfunction shows a high correlation with cardiovascular risk factors such as smoking, diabetes, hypertension and dyslipidaemia. ${ }^{12}$ Metabolic syndrome and central abdominal obesity are independent prognostic factors of penile erectile dysfunction. Individuals over 50 years of age suffering from metabolic syndrome have a greater likelihood of having moderate to severe erectile dysfunction than mild erectile dysfunction. ${ }^{13}$

Many studies have evaluated the clinical aspects of metabolic syndrome, but studies on the pharmacologic effects on a model of induced metabolic syndrome are very rare. Matsuzaki et al. ${ }^{14}$ reported the pharmacological effects of blood pressure-reducing agents using OLETF rats with induced metabolic syndrome. Renna et al. ${ }^{15}$ reported the long-term

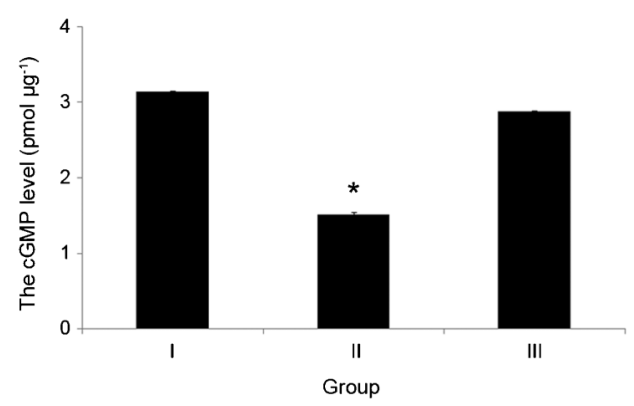

Figure 5 The cGMP level of the corpus cavernosum in each group. The cGMP level in group II was significantly lower than that of the control group $(* P=0.005$ vs. group I). cGMP, cyclic guanosine monophosphate. Group I, control; Group II: metabolic syndrome + normal saline; Group III: metabolic syndrome + Korean red ginseng. effects of aspirin-containing agents administered to rats with metabolic syndrome induced by a high-fructose diet. In the field of urology, Filippi et al. ${ }^{16}$ have developed a new animal model of metabolic syndrome associated with hypogonadotropic hypogonadism and erectile dysfunction induced by feeding rabbits a high-fat diet. Using the same rabbit model, Vignozzi et al. ${ }^{17}$ suggested that testosterone has a beneficial effect on prostate health, counteracting metabolic syndrome-associated prostate alterations, such as inflammation, hypoxia and fibrosis, which are key components in the development and progression of benign prostatic hyperplasia/lower urinary tract symptom. Additionally, Morelli et al. ${ }^{18}$ also demonstrated that the development of metabolic syndrome features in a high-fat diet rabbit model is associated with alterations not only of the prostate but also bladder derangements. Lee et al. ${ }^{19}$ reported on receptors associated with the symptoms of overactive bladder in rats with metabolic syndrome induced by a high-fructose diet. Two reports are similar to our study and were designed using an animal model of metabolic syndrome. The study by Cao et al. ${ }^{20}$ was designed to examine the effect of a high-fat diet on the cardiovascular structure and function in SHR rats, and Nagatomo et al. ${ }^{21}$ showed that a high-fat diet reduced oxidative capacity in skeletal muscles and accelerated metabolic syndrome using gene manipulation in SHR rats (SHR/NDmcr-cp rats). Regarding studies of the pharmacological effects, Anraku et al. ${ }^{22}$ reported antioxidant and antilipid effects of kitosan on SHR/ND mcrcp rats with spontaneous hypertension and modified leptin genes after inducing metabolic syndrome.

Because metabolic syndrome affects the corpus cavernosum penis vascular endothelial cells in the same way that atherosclerosis is induced in cardiac vessels, erectile dysfunction can be a good marker for identifying coronary heart disease. This is because atherosclerosis initially develops in the penis in blood vessels with a small diameter, resulting in erectile dysfunction. The risk of developing atherosclerosis in blood vessels with a large diameter is then increased. ${ }^{23}$ In addition, atherosclerosis contributes to structural damage in tissues of the corpus cavernosum penis, resulting in endothelial cell dysfunction. For instance, high blood glucose facilitates the production of reactive oxygen species, such as superoxide anion, to inactivate NO and increases free radicals, which activate protein kinase $\mathrm{C}$ and various cellular factors. Endothelial cell dysfunction reduces the NO level in the blood vessels and increases the concentration of free radicals, which are toxic and induce atherosclerotic damage. NO interferes with the adhesion of platelets and white blood cells to the blood vessel wall and reduces smooth muscle cell proliferation. ${ }^{9}$ High blood glucose induces the glycation of the corpus cavernosum penis and inhibits the regeneration of collagen fibres. Accordingly, erectile dysfunction can occur.

When KRG that has been steamed and then dried, this is more effective and has fewer side effect, according to body constitution have been reported in studies of oriental medicine. In addition, treatment effects are known to be enhanced in the process of steaming and drying because specific ginsenoside ingredients are removed or generated. According to in vivo animal experiments, KRG affects NO, calcium and potassium channels and relaxes the smooth muscles of the corpus cavernosum penis. In particular, the saponin components of KRG relax the smooth muscles of the corpus cavernosum penis and increase the internal pressure. As a result, KRG enhances the erectile function of the penis, and the effects vary in accordance with the composition of the administered KRG. ${ }^{24}$ de Andrade et al. ${ }^{25}$ examined the treatment efficacy of KRG in impotent men with ED, and showed that KRG can be an effective alternative to invasive approaches for treating male ED. Kim et al. ${ }^{26}$ have reported the significant improvement of erectile function in patients treated with tissue-cultured mountain ginseng extract. 
The smooth muscles of the corpus cavernosum penis play an important role in the erection and relaxation of the penis. In normal adults, the ratio of smooth muscle cells in the corpus cavernosum penis is approximately $40 \%-52 \%$, while the ratio in vasogenic erectile dysfunction patients is decreased to $10 \%-36 \%$, confirming the progression of fibrosis in the corpus cavernosum penis, which is thought to be mainly due to the chronic accumulation of collagen caused by ischaemic smooth muscles of the corpus cavernosum penis and the accumulation of extracellular matrix proteins. TGF- $\beta_{1}$ plays an important role in these processes by aggregating fibroblasts at the area of ischaemic tissue damage and promoting the generation of collagenous connective tissues to induce the repair of damaged tissues. ${ }^{27}$ In SHR rats, Ushiyama et al. ${ }^{28}$ discovered that fibrosis of the corpus cavernosum penis was caused by the disposition of extracellular materials on interstitial tissues and nervous tissues around the smooth muscles of the corpus cavernosum penis. Park et al. ${ }^{29}$ investigated TGF- $\beta_{1}$ expression as a molecular marker of fibrosis in vascular erectile dysfunction, and they experimentally demonstrated that an increased level of TGF- $\beta_{1}$ resulted in increased fibrosis in the corpus cavernosum penis. Similarly, in our study, the ratio of smooth muscle cells was significantly lower in group II, which had induced metabolic syndrome, while the ratio of smooth muscle cells in group III, which was administered KRG, was similar to that of group I, suggesting the recovery of penile tissue with a reduction of fibrosis in the corpus cavernosum penis. As a result of investigating the correlation between TGF- $\beta_{1}$ expression and fibrosis after immunohistochemical staining, group II showed an abrupt increase in the number of collagen fibres and collagenous connective tissue with fibroblast aggregation around the cavernosus and demonstrated high TGF- $\beta_{1}$ immunoreactivity. By contrast, group III showed significantly reduced reactivity, demonstrating the effects of KRG, which reversibly improved fibrosis in the corpus cavernosum penis.

The definition of metabolic syndrome in the rat model has not been confirmed, and metabolic syndrome data from animal models are lacking. We attempted to create an animal model of metabolic syndrome. This is the first study to determine the effects of Korean red ginseng extract on erectile function in a rat model of metabolic syndrome.

In the present study, KRG improved erectile function in a rat model of metabolic syndrome. KRG inhibited the fibrosis of the corpus cavernosum penis. This result demonstrates that KRG can be used as an alternative medicine to improve metabolic syndrome and recover penile erectile function in patients with metabolic syndrome and erectile dysfunction.

\section{AUTHOR CONTRIBUTIONS}

SDK had performed drafting the manuscript, conception and design, acquisition of data. YJK carried out the statistical analysis. JSH participated in the design of the study and helped to draft the manuscript. SWK participated in the sequence alignment. DWS made animal model conception and designed conception and design, analysis and interpretation of data.

\section{COMPETING FINANCIAL INTERESTS}

The authors declare no competing financial interests.

\section{ACKNOWLEDGMENTS}

This study was supported in 2009 by the Jeju National University Hospital research Foundation (No. CNUH 2009-05).

1 Pegge NC, Twomey AM, Vaughton K, Gravenor MB, Ramsey MW et al. The role of endothelial dysfunction in the pathophysiology of erectile dysfunction in diabetes and in determining response to treatment. Diabet Med 2006; 23: 873-8.
2 Ferrini MG, Kovanecz I, Sanchez S, Vernet D, Davila HH et al. Long-term continuous treatment with sildenafil ameliorates aging-related erectile dysfunction and the underlying corporal fibrosis in the rat. Biol Reprod 2007; 76: 915-23.

3 Esposito K, Giugliano F, Martedi E, Feola G, Marfella R et al. High proportions of erectile dysfunction in men with the metabolic syndrome. Diabetes Care 2005; 28: 1201-3.

4 Kim SC. Insulin resistance and erectile dysfunction. Korean J Urol 2006; 47: 917-27

5 Abate N, Chandalia M, Cabo-Chan AV Jr, Moe OW, Sakhaee K. The metabolic syndrome and uric acid nephrolithiasis: novel features of renal manifestation of insulin resistance. Kidney Int 2004; 65: 386-92.

6 Lund Håheim L, Wisløff TF, Holme I, Nafstad P. Metabolic syndrome predicts prostate cancer in a cohort of middle-aged Norwegian men followed for 27 years. Am J Epidemiol 2006; 164: 769-74.

7 Kim SD, Cho SY, Sohn DW, Lee DS, Jeong MS et al. [The changes of the erectile function and histology of the corpus cavernosum in a rat model of metabolic syndrome]. Korean J Androl 2008; 26: 187-96.

$8 \mathrm{Ko} \mathrm{SH}$, Kwon HS, Kim SR, Moon SD, Ahn YB et al. Ramipril treatment suppresses islet fibrosis in otsuka long-evans tokushima fatty rats. Biochem Biophys Res Commun 2004; 316: 114-22.

9 Lee RK, Chughtai B, Te AE, Kaplan SA. Sexual function in men with metabolic syndrome. Urol Clin North Am 2012; 39: 53-62.

10 Corona G, Mannucci E, Ricca V, Lotti F, Boddi V et al. The age-related decline of testosterone is associated with different specific symptoms and signs in patients with sexual dysfunction. Int J Androl 2009; 32: 720-8.

11 Chughtai B, Lee RK, Te AE, Kaplan SA. Metabolic syndrome and sexual dysfunction. Curr Opin Urol 2011; 21: 514-8.

12 Feldman HA, Johannes CB, Derby CA, Kleinman KP, Mohr BA et al. Erectile dysfunction and coronary risk factors: prospective results from the Massachusetts male aging study. Prev Med 2000; 30: 328-38.

13 Heidler S, Temml C, Broessner C, Mock K, Rauchenwald M et al. Is the metabolic syndrome an independent risk factor for erectile dysfunction? J Urol 2007; 177: 651-4.

14 Matsuzaki G, Ishizaka N, Furuta K, Hongo M, Saito K et al. Comparison of vasculoprotective effects of benidipine and losartan in a rat model of metabolic syndrome. Eur J Pharmacol 2008; 587: 237-42.

15 Renna NF, Vazquez MA, Lama MC, Gonzalez ES, Miatello RM. Effect of chronic aspirin administration on an experimental model of metabolic syndrome. Clin Exp Pharmaco Physiol 2009; 36: 162-68.

16 Filippi S, Vignozzi L, Morelli A, Chavalmane AK, Sarchielli E et al. Testosterone partially ameliorates metabolic profile and erectile responsiveness to PDE5 inhibitors in an animal model of male metabolic syndrome. J Sex Med 2009; 6: 3274-88.

17 Vignozzi L, Morelli A, Sarchielli E, Comeglio P, Filippi S et al. Testosterone protects from metabolic syndrome-associated prostate inflammation: an experimental study in rabbit. J Endocrinol 2012; 212: 71-84.

18 Morelli A, Comeglio P, Filippi S, Sarchielli E, Cellai I et al. Testosterone and farnesoid $X$ receptor agonist INT-747 counteract high fat diet-induced bladder alterations in a rabbit model of metabolic syndrome. J Steroid Biochem Mol Biol 2012; 132: 80-92.

19 Lee WC, Chuang YC, Chiang PH, Chien CT, Yu HJ et al. Pathophysiological studies of overactive bladder and bladder motor dysfunction in a rat model of metabolic syndrome. J Urol 2011; 186: 318-25

20 Cao J, Sodhi K, Puri N, Monu SR, Rezzani R et al. High fat diet enhances cardiac abnormalities in SHR rats: protective role of heme oxygenase-adiponectin axis. Diabetol Metab Syndr 2011; 3: 37

21 Nagatomo F, Fujino H, Kondo H, Takeda I, Tsuda K et al. High-fat diet-induced reduction of peroxisome proliferator-activated receptor- $\gamma$ coactivator- $1 \alpha$ messenge RNA levels and oxidative capacity in the soleus muscle of rats with metabolic syndrome. Nutr Res 2012; 32: 144-51.

22 Anraku M, Michihara A, Yasufuku T, Akasaki K, Tsuchiya D et al. The antioxidative and antilipidemic effects of different molecular weight chitosans in metabolic syndrome model rats. Biol Pharm Bull 2010; 33: 1994-8.

23 Blumentals WA, Gomez-Caminero A, Joo S, Vannappagari V. Should erectile dysfunction be considered as a marker for acute myocardial infarction? Results from a retrospective cohort study. Int J Impot Res 2004; 16: 350-3.

24 Choi Y, Park J, Choi H, Nam K. [Effects of composition of saponin fraction from Korean red ginseng in the relaxation of rabbit and rat corpus cavernosum]. J Ginseng Res 1999; 23: 13-20.

25 de Andrade E, de Mesquita AA, Claro Jde A, de Andrade PM, Ortiz V et al. Study of the efficacy of Korean Red Ginseng in the treatment of erectile dysfunction. Asian J Androl 2007; 9: 241-4.

$26 \mathrm{Kim} \mathrm{TH}$, Jeon SH, Hahn EJ, Paek KY, Park JK et al. Effects of tissue-cultured mountain ginseng (Panax ginseng CA Meyer) extract on male patients with erectile dysfunction. Asian J Androl 2009; 11: 356-61.

27 Nehra A, Goldstein I, Pabby A, Nugent M, Huang YH et al. Mechanisms of venous leakage: a prospective clinicopathological correlation of corporeal function and structure. J Urol 1996; 156: 1320-9.

28 Ushiyama M, Morita T, Kuramochi T, Yagi S, Katayama S. Erectile dysfunction in hypertensive rats results from impairment of the relaxation evoked by neurogenic carbon monoxide and nitric oxide. Hypertens Res 2004; 27: 253-61.

29 Park K, Shin JW, Jeong SJ, Park DW, Son $\mathrm{H}$ et al. [Pathophysiologic investigation of vasculogenic erectile dysfunction using newly developed rat model]. Korean J Androl 2003; $21: 136-44$. 